Cite this: Phys. Chem. Chem. Phys., 2011, 13, 7747-7750

\title{
DMF-exfoliated graphene for electrochemical NADH detection
}

\author{
Gareth P. Keeley, ${ }^{* a b c}$ Arlene O'Neill, ${ }^{b d}$ Michael Holzinger, ${ }^{a}$ Serge Cosnier, ${ }^{a}$ \\ Jonathan N. Coleman ${ }^{b d}$ and Georg S. Duesberg ${ }^{b c}$
}

Received 7th January 2011, Accepted 7th March 2011

DOI: $10.1039 / \mathrm{c} 1 \mathrm{cp} 20060 \mathrm{~g}$

The electrochemical detection of NADH is of considerable interest because it is required as a cofactor in a large number of dehydrogenase-based biosensors. However, the presence of oxygenated functionalities on the electrode often causes fouling due to the adsorption of the oxidised form, $\mathrm{NAD}^{+}$. Here we report an electroanalytical NADH sensor based on DMF-exfoliated graphene. The latter is shown to have a very low oxygen content, facilitating the exceptionally stable and sensitive detection of this important analyte.

\section{Introduction}

Carbon materials are widely used in electrochemistry due to their low cost, wide potential window and electro-catalytic activity for a range of redox reactions. ${ }^{1}$ The electrochemistry of 'traditional' $\mathrm{sp}^{2}$-hybridised carbon materials such as glassy carbon and highly-ordered pyrolytic graphite (HOPG) is well established, and the last decade or so has seen an extraordinary amount of research into the electrochemical characteristics of carbon nanotubes. It has been shown that the latter are endowed with excellent electron transfer properties, but the reliable interpretation of these is hampered by the various (and often unspecified) amounts of structural defects, metallic impurities and surface functionalities found in nanotubes. ${ }^{2}$ Graphene, the newest member of the carbon family, is now attracting interest as an electrode material. Initial reports of graphene synthesis focussed on mechanical exfoliation, from which the yields are too low for the fabrication of electrochemical electrodes. This is why the first study of graphene's electrochemical behaviour did not appear until 2008, when Shang et al. $^{3}$ used microwave plasma-enhanced chemical vapour deposition to grow vertically aligned graphene nanosheets (GNSs) on Si substrates. Today, several methods are known to produce high-yield graphene, and there are already a number of reviews covering the emergence of this remarkable material in electrochemistry. ${ }^{4-7}$ The present work involves graphene nano-sheets produced using the liquid-phase

\footnotetext{
a Département de Chimie Moléculaire, UMR 5250, ICMG FR 2607,

CNRS, Université Joseph Fourier, BP 53, 38041 Grenoble cedex 9,

France. E-mail: gkeeley@ujf-grenoble.fr; Fax: + 3345652 0805;

Tel: +33456520853

${ }^{b}$ Centre for Research on Adaptive Nanostructures and Nanodevices

(CRANN), Dublin 2, Ireland

${ }^{c}$ School of Chemistry, Trinity College, Dublin 2, Ireland

${ }^{d}$ School of Physics, Trinity College, Dublin 2, Ireland
}

exfoliation of graphite. ${ }^{8,9}$ This approach lends itself particularly well to the creation of electrode materials because it does not proceed via a catalytic deposition or graphitic oxide intermediate, so contributions from metallic impurities and residual oxides towards electro-catalysis may be safely ruled out. ${ }^{9}$

The electrochemical oxidation of $\beta$-nicotinamide adenine dinucleotide (NADH) is of considerable interest because it is required as a cofactor in a large number of dehydrogenasebased biosensors. ${ }^{10}$ Many different electrode systems have been investigated as NADH sensors, ${ }^{11}$ including reduced graphene oxide $(\mathrm{GO})^{12-14}$ and ionic liquid-functionalised graphene. ${ }^{15}$ Efforts are often hindered by the need for large overpotentials $(\mathrm{ca} .>+0.5 \mathrm{~V})$, as well as electrode fouling due to the adsorption of the oxidised form, $\mathrm{NAD}^{+}$. In this communication, the application of graphene produced from liquid-phase exfoliation as an electrode material for NADH oxidation is presented for the first time. The sensor is shown to exhibit features which compare favourably with various systems previously reported. In particular, the low oxygen content of this graphene provides a fouling resistance superior to sensors based on reduced graphene oxide. Edge-plane sites and defects on the graphene surface are identified as the promoters of electron transfer, and comparisons with GO are used to demonstrate the unique advantages offered by graphene produced in this way.

\section{Experimental}

Graphite powder (product number 332461), $\beta$-nicotinamide adenine dinucleotide (N4505, reduced dipotassium salt), ammonium iron(III) sulfate, monobasic sodium phosphate and dibasic sodium phosphate were purchased from SigmaAldrich. All solutions were prepared using water (resistivity $18.2 \mathrm{M} \Omega \mathrm{cm}$ ). The $0.1 \mathrm{M}, \mathrm{pH} 6.9$ phosphate buffer solution was prepared by dissolving mono- and dibasic sodium phosphate in water in the appropriate ratio. An Autolab PGSTAT30 potentiostat was used to perform electrochemical measurements, along with a three-electrode configuration. IJ Cambria supplied glassy carbon working electrodes (CHI104, diameter $3 \mathrm{~mm}$ ), $\mathrm{Ag} / \mathrm{AgCl}$ reference electrodes (CHI11) and Pt wire counter electrodes (CHI115). Electrolytes were purged for $20 \mathrm{~min}$ using high-purity Ar before experiments, and a blanket of Ar was maintained over the solution during measurements. All of the latter were performed at room temperature. For chronoamperometric measurements, 
stirring $(260 \mathrm{rpm})$ was provided by a magnetic bar. Differential pulse voltammetry was carried out using a pulse modulation of $+50 \mathrm{mV}$, a duration of $50 \mathrm{~ms}$ and a period of $0.5 \mathrm{~s}$.

A comprehensive description of the graphene fabrication procedure, along with a thorough morphological and electrochemical characterisation of the material, can be found in our previous work. ${ }^{9}$ In short, the nano-sheets are produced by the dispersion and prolonged sonication of graphite flakes in DMF $\left(3.3 \mathrm{mg} \mathrm{cm}^{-3}\right)$. They are typically sub-micron in lateral size and composed of five or fewer graphitic layers. The GNSs have a large electrochemical surface area and a very low oxygen content. The pre-modification polishing procedure for the glassy carbon electrodes has also been described previously. ${ }^{16}$ In order to prepare GC/GNS electrodes, graphene suspensions were dropped $(9 \mu \mathrm{L})$ directly onto inverted glassy carbon electrodes and the solvent was allowed to dry. The resulting GNS films were clearly visible to the naked eye. The modified electrodes were rinsed sequentially with deionised water and working electrolyte before being placed in the cell.

\section{Results}

Preliminary investigations regarding the effect of GNS modification on the response of glassy carbon electrodes to NADH were conducted using cyclic voltammetry. Fig. 1(a) shows a typical comparison between bare and modified electrodes. Peak currents at the latter are increased due to the large electrochemically active surface area of the graphene. ${ }^{9}$ Moreover, the oxidation peak potential of $0.39 \mathrm{~V}(v s . \mathrm{Ag} / \mathrm{AgCl})$ is some $0.13 \mathrm{~V}$ lower than that observed at the unadulterated electrode, indicating a possible electro-catalytic effect. The latter is attributed to the high proportion of edge-plane sites and defects present in the graphene nano-sheets, due to their small lateral size. ${ }^{9}$ Banks and Compton ${ }^{17}$ have previously identified these regions as promoters of NADH oxidation at HOPG electrodes. An interesting corollary to this interpretation is that, since graphene electrochemistry is dominated by edge regions, there is no particular advantage in using monolayer graphene instead of few- or multi-layer graphene as an electrode material, as $\mathrm{we}^{9}$ and others ${ }^{18}$ have recently suggested. Rather, it is recommended that efforts should focus on the reduction of lateral flake size and not flake thickness.

The graphene-based NADH sensor was probed further using chronoamperometry. Initially, only phosphate buffer $\left(10 \mathrm{~cm}^{3}\right)$ was present in the cell. Stirring was initiated, the electrode was poised at $+0.4 \mathrm{~V}(v s . \mathrm{Ag} / \mathrm{AgCl})$ and aliquots $(50 \mu \mathrm{L})$ of stock NADH solution were added at $50 \mathrm{~s}$ intervals. Fig. 1(b) shows the rapid response of the modified electrode compared to the lethargic response of bare glassy carbon at this operating potential. The GC/GNS electrodes were found to exhibit linear responses to additions of NADH up to a concentration of $360 \mu \mathrm{M}$ (eight additions of $45 \mu \mathrm{M}$ ), after which the noise generated by stirring precluded the unambiguous measurement of the current. Similar problems with this technique have been previously reported in relation to the study of nitrite oxidation at bare glassy carbon electrodes. ${ }^{19}$ The inset of Fig. 1(b) shows a plot of current against concentration over the measurable range (correlation coefficient $0.996, N=8$ ).
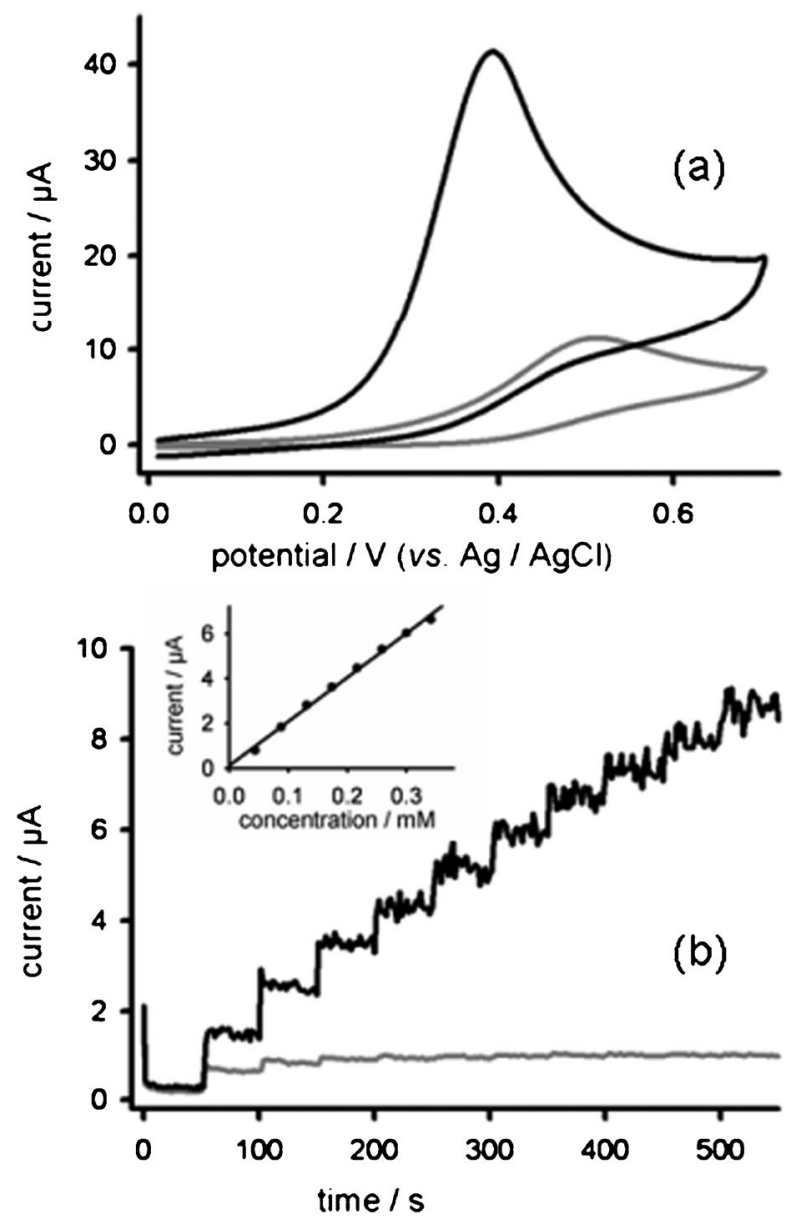

Fig. 1 (a) Cyclic voltammograms recorded at bare (grey) and GNSmodified (black) GCEs in $1 \mathrm{mM}$ NADH in $0.1 \mathrm{M}$ phosphate buffer ( $\mathrm{pH}$ 6.9) at a sweep rate of $100 \mathrm{mV} \mathrm{s}^{-1}$. (b) Chronoamperometric recordings obtained at $+0.4 \mathrm{~V}(v s . \mathrm{Ag} / \mathrm{AgCl})$ on increasing the NADH concentration in known steps at these electrodes. The inset shows the resulting plot of steady-state current against NADH concentration for the modified electrode.

The sensitivity, $b$, was found to be $19.7 \mathrm{~mA} \mathrm{M}^{-1}$. In order to calculate the detection limit, ten blank measurements were performed in phosphate buffer containing no NADH. The standard deviation, $\sigma$, of these was $0.0124 \mu \mathrm{A}$. Using the formula $3 \sigma / b$, the detection limit was found to be $1.9 \mu \mathrm{M}$. This approach to the calculation is similar to work reported by Zhou et al. ${ }^{20}$ The latter is a rare example of an unambiguous description of the calculation of this important parameter. The minimum reliably measurable concentration (based on a signalto-noise ratio of 3) using chronoamperometry was $15 \mu \mathrm{M}$.

Chronoamperometry was also used to show that the GNS-modified electrodes exhibit a stable amperometric response. Before doing so, graphene oxide electrodes were fabricated by cycling GC/GNS electrodes fifty times in $0.2 \mathrm{M} \mathrm{HClO}_{4}$ between -0.5 and $+1.5 \mathrm{~V}$ at a sweep rate of $200 \mathrm{mV} \mathrm{s}^{-1}$. The resulting increase in surface oxygen content was confirmed using the $\mathrm{Fe}^{3+/ 2+}$ probe, whose kinetics are known to be strongly influenced by the presence of surface oxides. ${ }^{1}$ Fig. 2(a) shows that the pristine GNS electrodes exhibit an extremely sluggish response to this probe, whereas the graphene oxide electrodes give rise to ideal voltammograms, 

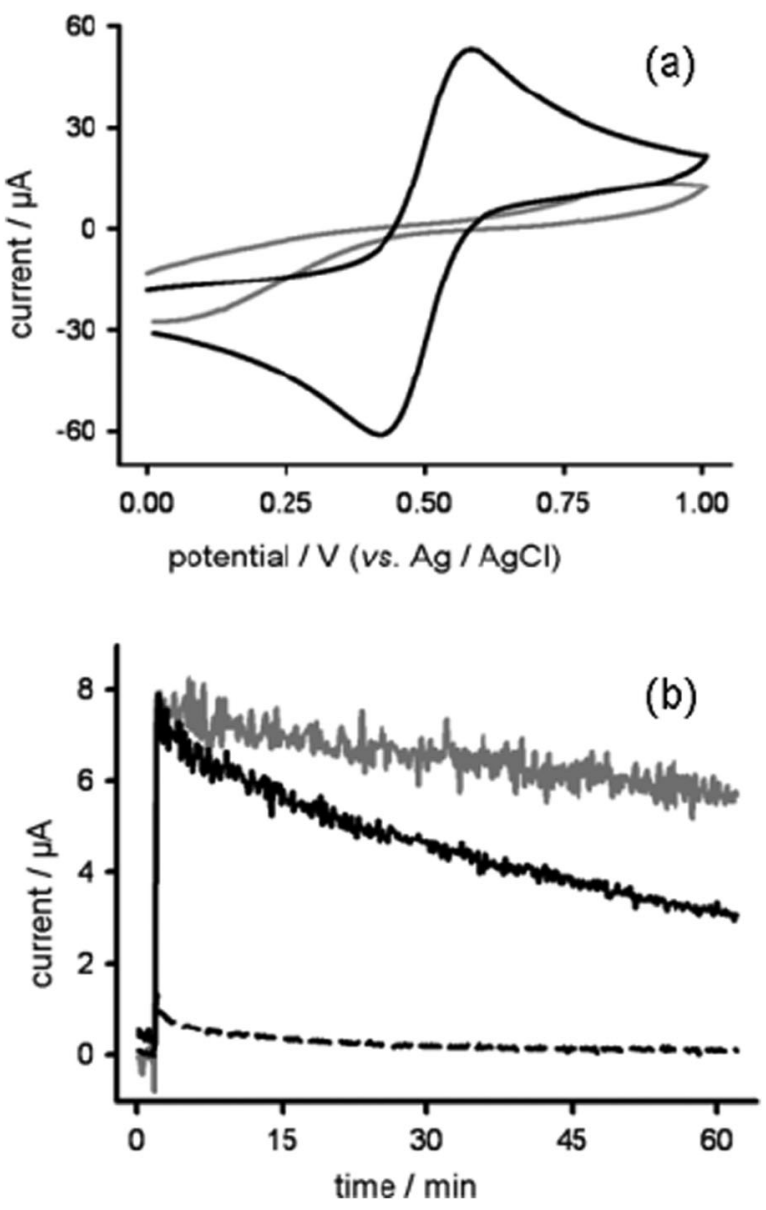

Fig. 2 (a) Voltammograms recorded at GC/GNS (grey) and GC/GONS (black) electrodes in $5 \mathrm{mM} \mathrm{Fe}^{3+/ 2+}$ in $0.2 \mathrm{M} \mathrm{HClO}_{4}$ at $100 \mathrm{mV} \mathrm{s}^{-1}$. (b) Chronoamperometric stability of the response to $0.4 \mathrm{mM}$ NADH for these electrodes. Also included is bare GC (dashed).

indicative of rapid electron transfer (ET). The stability of the $\mathrm{NADH}$ responses of the various electrode systems was then tested using chronoamperometry, and a typical result is shown in Fig. 2(b). Buffer was placed in the cell and the potential was fixed at $+0.4 \mathrm{~V}$ (vs. $\mathrm{Ag} / \mathrm{AgCl}$ ). After allowing the current to stabilise for $2 \mathrm{~min}$, NADH was added such that the concentration became $0.4 \mathrm{mM}$. The current increased as a result and was allowed to continue for one hour. During this time, the GC/GONS signal decayed to $37 \%$ of its initial value, while the pristine graphene electrode maintained $85 \%$ of its original signal. This indicates that modification of electrodes using liquid-phase exfoliated graphene provides impressive resistance to surface fouling. Graphene produced in this way is largely free of oxygenated functionalities ${ }^{8,9}$ which would facilitate the adsorption of the oxidised form. ${ }^{21,22}$ This represents a clear advantage of this material over graphene made via graphitic oxide intermediates for applications in dehydrogenase-based biosensors. Moreover, the enhanced ET kinetics and lower oxidation potential further limit the formation of radical intermediates. $^{23}$

Given the limitations of chronoamperometry described above, differential pulse voltammetry was used to further investigate the ability of graphene-modified electrodes to act as sensors for NADH. Fig. 3(a) shows a comparison between scans obtained using bare and modified electrodes. Again, it is clear that the presence of graphene gives rise to enhanced peak currents and lower oxidation potentials. The modified electrodes were used to detect NADH at a range of concentrations. Selected results are displayed in Fig. 3(b), and the inset shows the corresponding calibration plot. Peak currents increased linearly (correlation coefficient 0.999, $N=11$ ) with $\mathrm{NADH}$ concentration up to $600 \mu \mathrm{M}$. The detection limit was found to be $1.4 \mu \mathrm{M}$, based on a standard deviation of $0.0102 \mu \mathrm{A}$ (ten blank measurements) and a sensitivity of $22.3 \mathrm{~mA} \mathrm{M}^{-1}$. The minimum reliably measurable concentration was $10 \mu \mathrm{M}$. These values are competitive with many NADH sensors previously reported. ${ }^{11}$ It is also interesting to note they are superior to those found using chronoamperometry. It is therefore recommended that DPV should always be explored in sensor studies of this type, particularly since chronoamperometry is not a realistic representation of the approach normally used in analytical procedure. ${ }^{19}$ It is acknowledged, however, that Zhu, Chen and Yang $^{24}$ have reported a lower detection limit and wider linear
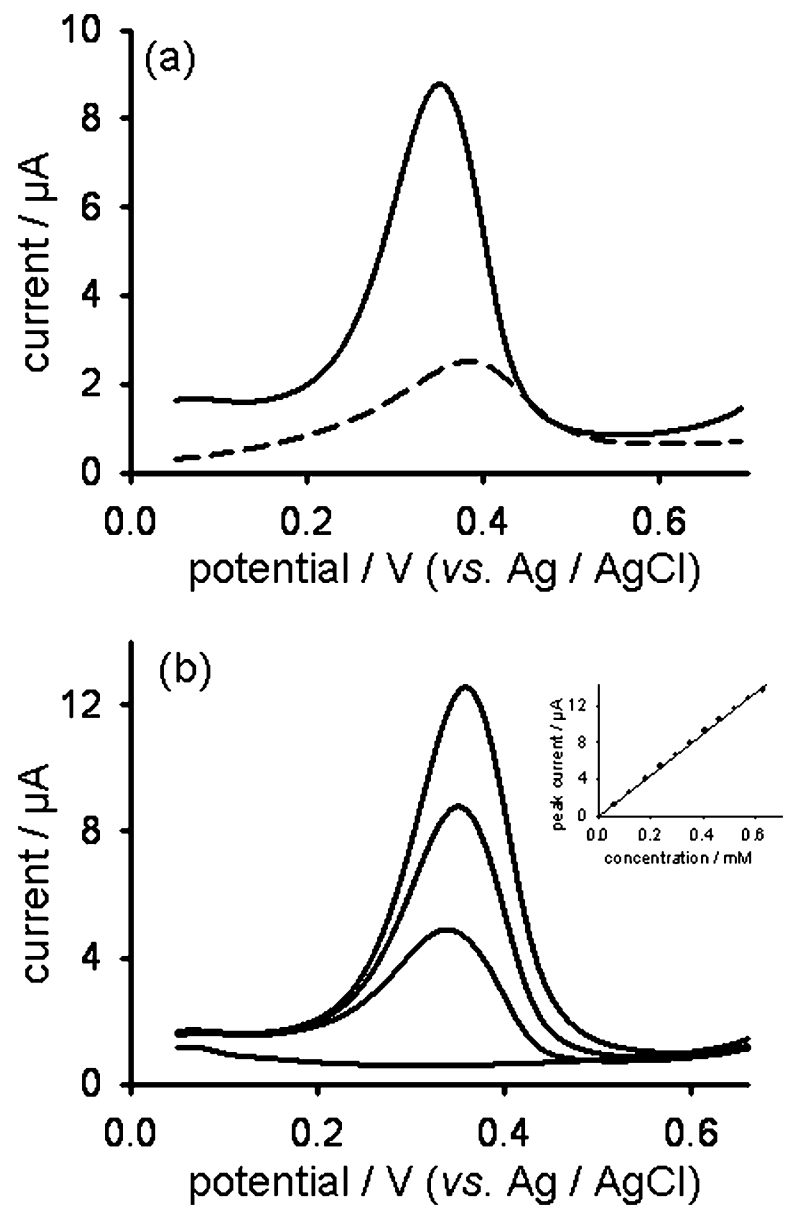

Fig. 3 (a) Differential pulse voltammograms recorded at bare (dashed) and GNS-modified (solid) GCEs in $0.35 \mathrm{mM}$ NADH in $0.1 \mathrm{M}$ buffer (pH 6.9). (b) Voltammograms recorded at a GC/GNS electrode in (from bottom to top) $0.00,0.18,0.35$ and $0.52 \mathrm{mM}$ $\mathrm{NADH}$. The inset shows the resulting plot of electro-catalytic peak current against NADH concentration. 
range using chronoamperometry at graphite nano-sheet modified electrodes.

\section{Conclusions}

For the first time, an electroanalytical sensor for NADH based on graphene produced using liquid-phase exfoliation has been demonstrated. The high coverage of edge-plane sites and defects shown by the graphene promotes the electro-oxidation of this important analyte, and its unfunctionalised surface avoids the fouling which commonly hampers carbon-based NADH sensors. The combination of exceptional stability and low operating potential is superior to previous reports involving graphene produced from the reduction of graphitic oxides. It is noteworthy that these advantages are inherent in the as-made material, and are not the result of surface treatment or modidication. The range, sensitivity and detection limit of the sensor compare favourably with values reported for other electrode systems. These findings constitute a significant step towards the incorporation of graphene into dehydrogenasebased amperometric biosensors.

\section{Acknowledgements}

G. P. K. is grateful for funding received from the Irish Research Council for Science, Engineering and Technology (IRCSET), co-funded by Marie Curie Actions under FP7. A. O'N. also thanks IRCSET for financial support. G. P. K., M. H. and S. C. acknowledge the platform 'Functionalisation of surfaces and transduction' of the scientific structure 'Nanobio' for the provision of facilities. The technical assistance of Mme. Arielle Le Pellec of l'Université Joseph Fourier is also warmly appreciated.

\section{Notes and references}

1 R. L. McCreery, Chem. Rev., 2008, 108, 2646.

2 I. Dumitrescu, P. R. Unwin and J. V. Macpherson, Chem. Commun., 2009, 6886.

3 N. G. Shang, P. Papakonstantinou, M. McMullan, M. Chu, A. Stamboulis, A. Potenza, S. S. Dhesi and H. Marchetto, Adv. Funct. Mater., 2008, 18, 3506.

4 M. Pumera, Chem. Rec., 2009, 9, 211.

5 Y. Shao, J. Wang, H. Wu, J. Liu, I. A. Aksay and J. Lin, Electroanalysis, 2010, 22, 1027.

6 D. A. C. Brownson and C. E. Banks, Analyst, 2010, 135, 2768.

7 D. Chen, L. Tang and J. Li, Chem. Soc. Rev., 2010, 39, 3157.

8 Y. Hernandez, V. Nicolosi, M. Lotya, F. M. Blighe, Z. Sun, S. De, I. T. McGovern, B. Holland, M. Byrne, Y. K. Gun'ko, J. J. Boland, P. Niraj, G. S. Duesberg, S. Krishnamurthy, R. Goodhue, J. Hutchison, V. Scardaci, A. C. Ferrari and J. N. Coleman, Nat. Nanotechnol., 2008, 3, 563.

9 G. P. Keeley, A. O'Neill, N. McEvoy, N. Peltekis, J. N. Coleman and G. S. Duesberg, J. Mater. Chem., 2010, 20, 7864.

10 T. N. Rao, I. Yagi, T. Miwa, D. A. Tryk and A. Fujishima, Anal. Chem., 1999, 71, 2506.

11 S. A. Kumar and S.-M. Chen, Sensors, 2008, 8, 739.

12 M. Zhou, Y. Zhai and S. Dong, Anal. Chem., 2009, 81, 5603.

13 J. Wang, S. Yang, D. Guo, P. Yu, D. Li, J. Ye and L. Mao, Electrochem. Commun., 2009, 11, 1892.

14 W.-J. Lin, C.-S. Liao, J.-H. Jhang and Y.-C. Tsai, Electrochem. Commun., 2009, 11, 2153.

15 C. Shan, H. Yang, D. Han, Q. Zhang, A. Ivaska and L. Niu, Biosens. Bioelectron., 2010, 25, 1504.

16 G. P. Keeley and M. E. G. Lyons, Int. J. Electrochem. Sci., 2009, 4 794.

17 C. E. Banks and R. G. Compton, Analyst, 2005, 130, 1232.

18 M. S. Goh and M. Pumera, Chem.-Asian J., 2010, 5, 2355.

19 B. R. Kozub, N. V. Rees and R. G. Compton, Sens. Actuators, B, 2010, 143, 539.

20 H. Zhou, Z. Zhang, P. Yu, L. Su, T. Ohsaka and L. Mao, Langmuir, 2010, 26, 6028.

21 J. Xu, Q. Chen and G. M. Swain, Anal. Chem., 1998, 70, 3146.

22 M. Pumera, R. Scipioni, H. Iwai, T. Ohno, Y. Miyahara and M. Boero, Chem.-Eur. J., 2009, 15, 10851.

23 L. Wu, X. Zhang and H. Ju, Anal. Chem., 2007, 79, 453.

24 J. Zhu, X. Chen and W. Yang, Sens. Actuators, B, 2010, 150, 564. 